\title{
Reconfiguración del sentido de enfermedad crónica: la emergencia de bienestar ${ }^{*}$
}

\section{Reconfiguration of the Meaning of Chronic Disease: The Emergence of Wellbeing}

\author{
Dora Isabel Garzón De Laverde** \\ ORCID: 0000-0002-2932-1794 \\ María Piedad Rojas Gil \\ ORCID: 0000-0002-8237-7156 \\ Universidad Santo Tomás. Bogotá \\ Elkin Higuera-Dagovett \\ ORCID: 0000-0002-2730-5731 \\ Universidad de Ciencias Aplicadas \\ y Ambientales UDCA
}

Recibido: 2 de septiembre de 2019 Revisado: 8 de noviembre de 2019 Aceptado: 30 de marzo de 2020

\section{Resumen}

Esta investigación pretende comprender cómo se configuran y reconfiguran en el relato los órdenes de sentido y acción para la emergencia del bienestar. Esto, mediante diálogos generativos en la relación que establecen pacientes con enfermedades crónicas en diferentes etapas de ciclo vital, su familia y profesionales de la salud. Se realizó esta investigación cualitativa, con diseño narrativo-conversacional. La experiencia de enfermedad crónica se organiza en historias centradas en problemas, con cristalización del sí mismo en la relación cuidador-paciente. Esto implica la construcción de identidad centrada en la imposibilidad de cuidar en un sentido totalizante que desdibuja la diversidad del sí mismo. En el contexto conversacional surgen nuevos sentidos de la enfermedad crónica, emergente en las diferentes formas de acción y de relación; lo que implica coevolución hacia formas de organización y afrontamiento generativo con nuevos posicionamientos del sí mismo centrado en la recuperación del poder que tienen las personas y las familias para decidir dentro de opciones posibles.

Palabras Clave: Enfermedad crónica, bienestar, relaciones familiares, sí mismo

Artículo de investigación. Citar como: Garzón, D. I., Rojas, G. M. P. y Higuera-Dagovett, E. (2020). Reconfiguración del sentido de enfermedad crónica: la emergencia de bienestar. Diversitas: Perspectivas en Psicología, 16(2), 441-454. https://doi.org/10.15332/22563067.6322

Autora de correspondencia: Dora Isabel Garzón De Laverde, Maestría en Psicología Clínica y de la Familia. Universidad Santo Tomás, Colombia. Dirección postal: Cra 9 No 51-11, Bogotá. Correo electrónico: doraisabelgarzon@hotmail.com 


\section{Abstract}

This research intends to gain insight into the pathways on which people narratives are configured and reconfigured to obtain the emergence of wellbeing. This is done through generative dialogues established by patients with chronic diseases at different stages of the life cycle, their families and health professionals. A qualitative research was carried out with a narrative-conversational design. Chronic disease experience is organized in problem-based stories and depicts a scenario where the self is crystallized in the relationship carer-patient, which implies the construction of an identity centered on the impossibility of taking care in a widespread sense that, in turn, fades the diversity of self. New insights on chronic disease experience have emerged during the conversational process, providing different meanings and relations. Therefore, it comprises a co-evolution towards novel ways of organization and generative coping, which enables new strategies to empower the patient and involve their relatives in a process of shared responsibilities aimed at improving the health status of people with chronic diseases.

Keywords: chronic disease, wellbeing, family relations, self.

\section{Introducción}

Para Krieger (2005) el contexto histórico y social en que viven los seres humanos se relaciona con la condición de salud-enfermedad de las personas. Así, las experiencias vividas desde la inequidad social pueden tener impacto en la dimensión biológica, pudiéndose inclinar la balanza hacia condiciones de enfermedad; esto es lo que Krieger define como embodiment (encarnación). Varela (2002) utiliza el término "encarnación" para hacer referencia a que lo que conocemos como realidad, solo es posible gracias a la interacción con los elementos que hacen parte de esta misma realidad. Para Varela (2002) existe una conexión entre lo biológico y el "Yo", como niveles de interacción que están co-determinados, a esta relación la define como emergencia de la consciencia del "si mismo" desde los biológico, concepto que se puede expandir a la "vida social consciente" (p.252), como una codeterminación Yo-Otro.

Así mismo, el construccionismo social con teóricos como Gergen (1996) y Anderson (1997) entre otros, sugieren la importancia de las conversaciones y de la narrativa en la construcción de la experiencia humana. En esa línea, Estupinán y González (2012), proponen que las personas relatan historias, las cuales, dan cuenta de los acontecimientos de la vida y de la experiencia, entendida esta última como el sentido y significado que adquieren esos acontecimientos. Estos relatos, que según White y Epston (1993), al estar saturados de problemas, limitan la vida de las personas, en este caso, centradas exclusivamente en la enfermedad sin concebir opciones de vida saludables donde sea posible la vivencia de bienestar.

Igualmente, en las conversaciones surgen relatos periféricos que no están suficientemente articulados con las historias dominantes que cristalizan la experiencia en lo problemático, los cuales pueden convertirse en nuevas versiones alejadas del problema y favorecen la construcción de relatos alternos (Estupinán y González, 2012; White y Epston, 1993). Estos relatos pueden centrarse en formas de afrontamiento generativo, en tanto, tienen el potencial de configurar estados de bienestar para los seres humanos. En resumen, estas propuestas comprenden los fenómenos humanos a partir de las formas de relación, el diálogo y la interacción que 
constituyen el significado de los acontecimientos en el día a día de las personas, organizadores de la experiencia y constructores de nuevas realidades.

Por lo tanto, se considera que la conversación es un proceso de generación de sentido y su capacidad transformacional, se apoya en su naturaleza dialógica y reflexiva, que posibilita narrar los sucesos de la vida y en ese mismo acto, encontrar sentidos diferentes, que son únicos y apropiados para la situación y las personas que participan en esta (Anderson, 1997). A su vez, la narrativa está contextualizada en la conversación, donde el relato adquiere órdenes de significado y acción en el encuentro de los seres humanos (Estupinán y González, 2012).

En este orden, es importante plantear el bienestar humano en pacientes con enfermedad crónica en la peculiaridad de las diferentes etapas de ciclo vital dentro de una perspectiva sistémico-compleja que tome en cuenta los sentidos y transformaciones posibles en relación al posicionamiento de pacientes, familias y profesionales del sistema de salud, que involucra, entre otros, la particularidad de los sistemas y su capacidad de gestión (Garzón y Rojas-Gil, 2016; Higuera-Dagovett, Rojas-Gil y Garzón, 2015; Higuera-Dagovett y Garzón, 2019). Por lo tanto, el propósito de la presente investigación implica comprender la forma como se configuran y se reconfiguran en el relato, los órdenes de sentido y acción que establecen pacientes con diagnóstico de enfermedades crónicas en diferentes etapas de ciclo vital, la familia y los profesionales de la salud, de tal forma que se facilite la emergencia de bienestar en procesos de diálogos generativos.

\section{Método}

Se realizó una investigación cualitativa de casos múltiples, contextual, con diseño narrativo conversacional y elementos de análisis del discurso. Adicional se realizó una investigación/intervención (Pakman, 1995), que consiste en generar procesos sincrónicos que permiten dar cuenta del cambio, dentro de un conocimiento que ha sido reflexionado y que transita desde los sistemas observados a los sistemas observantes, y es definido como epistemología de segundo orden (Von Foerster, 1996).
Para la selección de los participantes, se realizó un muestreo intencional con los siguientes criterios: 1 ) diagnóstico de enfermedad crónica y 2) aceptación voluntaria de las personas a participar (consentimiento y asentimiento informados). Participaron 19 personas: cuatro pacientes en momentos de ciclo vital: infancia, adolescencia, adulto y adulto mayor; dos madres, dos hijas, dos nietos y nueve profesionales, incluidos siete médicos, una enfermera y una auxiliar de información, de los servicios de hospitalización y consulta externa de un Hospital de ॥ nivel de la ciudad de Bogotá D.C. Colombia.

A su vez, se realizaron encuentros dialógico generativos, que de acuerdo a la propuesta de Fried Schitman, (2010a, 2010b, 2013, 2015), permiten asumir los dilemas y las experiencias, así como las crisis humanas desde las fortalezas y potencialidades de las personas y grupos, lo cual se constituye en posibilidad para la generación de redes de recursos internos y externos, promoción de la creatividad personal y social y la configuración de futuros posibles y realizables, los cuales conllevan aprendizajes y transformaciones, donde el sí mismo adquiere reconocimientos y renovaciones.

En el caso del niño, el encuentro se desarrolló con la madre y su hijo en el salón de juegos del hospital, junto con una auxiliar de información. Con la adulta mayor, por su estado crítico, la conversación se realizó con la hija y los nietos, seguida del encuentro con la médica interna. El adulto participó con su hija adolescente, y el joven, con su madre. El encuentro con profesionales se llevó a cabo a partir de la presentación de los resultados obtenidos en los encuentros con los pacientes y las familias.

Las conversaciones fueron grabadas en audio, se transcribieron y la información fue organizada en matrices. Se trabajó con los conceptos de codificación abierta (fase descriptiva) y codificación axial (fase interpretativa) de la Teoría Fundamentada (Corbin y Strauss, 2010) para la construcción de las categorías de análisis: 1) emergencia de bienestar en el contexto de salud en la relación pacientefamilia-equipo profesional, con las subcategorías emergentes: a) Acoples relacionales de los miembros de la familia y del sistema de salud alrededor del cuidado; y b) Procesos de subjetivación. Estas 
asociadas a la categoría transversal de todo el estudio de "experiencia de salud-enfermedad crónica en diferentes momentos de ciclo vital". Categorías que se cruzaron con los conceptos narrativos de: Historias, Memorias y Relatos Alternos (Estupinán y González, 2012; White y Epston, 1993), que permitieron dar cuenta del cambio construido en el proceso conversacional.

Se presentó en los ciclos: infancia, adolescencia, adulto y adulto mayor en su dinamismo y co-evolución (Kauffman, 2003), entendida la co-evolución como el proceso donde todos los integrantes del contexto evolucionan de manera conjunta en relación con el mismo entorno que crean. Así mismo, este entorno los alberga de manera armónica con diversidad de sentidos, en conexión con los diferentes ciclos vitales que dan cuenta de particularidades en la experiencia de salud-enfermedad crónica de acuerdo al momento vital asociado a los significados de la cultura y de la sociedad, donde se configuran procesos adaptativos y de supervivencia. Con lo anterior, la salud-enfermedad da cuenta del carácter singular de la experiencia en familias y pacientes de acuerdo a su momento vital en sus relaciones con el sistema de salud y contextos socio-culturales e institucionales, todos centrados en la consecución de bienestar.

\section{Resultados}

Los resultados se organizaron en las categorías de historias, centradas en el problema desde donde se posicionan y viven el acontecimiento de la enfermedad crónica las voces narradoras. Luego se da cuenta de las memorias, o relatos que no estaban articulados con la historia, pero que emergen para dar curso a los relatos alternos que refieren la reconfiguración de la experiencia.

\section{Ciclo Infancia}

Lactante de 18 meses de edad, quien desde los 15 días de nacido presentó manifestaciones clínicas respiratorias, hospitalizado en nueve ocasiones con diagnóstico de "síndrome bronco-obstructivo sobre infectado y bronco-neumopatía crónica”. La madre de 22 años es la cuidadora, tiene otros dos hijos con su pareja, cuya custodia y protección la tiene su madre por disposición del Instituto Colombiano de Bienestar Familiar (ICBF), institución dedicada a la protección integral de la infancia.

\section{Emergencia de bienestar en el contexto de salud en la relación paciente-familia-equipo profesional}

\section{Acoples relacionales de los miembros de la familia y del sistema de salud alrededor del cuidado}

Historias. Las múltiples consultas a los servicios de urgencias y hospitalización dan cuenta de una relación de dependencia cuidador-paciente-sistema de salud. Los profesionales no entienden y se sienten agotados pues sus prescripciones no surten el efecto esperado.

[...] "tenemos un equipo que hace un seguimiento bueno y no es para que ese niño acuda con tanta frecuencia y no es para que salga de aquí y se enferme nuevamente, entonces ¿Qué está pasando con ese entorno? ¿Qué está pasando con ese niño?" [...] otra vez esta señora acá..." 19-CIN-DJ.

La madre relata como las dinámicas familiares se configuran desde su responsabilidad exclusiva en el cuidado del hijo, lo que favorecen vínculos conflictivos en la relación de pareja, asociados al escaso tiempo compartido y la falta de apoyo del padre en las hospitalizaciones. El padre culpabiliza al hijo por la falta de tiempo de la madre para los demás miembros de la familia, y a la hija mayor por el alejamiento de su familia extensa, dada una situación de abuso sexual a la niña propiciado por un familiar suyo y las consecuencias legales por este hecho.

Memorias. Ante las nuevas comprensiones de la auxiliar de información al escuchar a la madre en su relato, refiere: no habían considerado en el Hospital que la madre pudiera tener tanto temor por la posible pérdida de su hijo.

Respecto a la pregunta de: ¿Cómo vive la experiencia con el ICBF?, la madre manifiesta que ha sido 
algo que ha servido para que los niños estén mejor cuidados pues su mamá le colabora muy bien; la relación demandante al sistema de salud es interpretada como una relación basada en la responsabilidad, pues ella "no descuida a su hijo como le paso con la niña”.

Relatos Alternos. En el seguimiento realizado siete meses más tarde, se encontró una mujer que manifiesta sentirse muy contenta pues encontró una forma propia de cuidar bien a sus hijos; al preguntarle: "Como está la familia y el niño?, responde: "en alguna ocasión en que estuvo agripado y con tos [...] yo le di remedios caseros que le sirvieron mucho, ya no he necesitado llevarlo al hospital", afirma que no ha necesitado volver a ningún hospital por la buena evolución de la salud del niño.

Manifiesta que se separó del padre de los niños. Vive con estos, su madre y su hermana: "las tres estamos muy tranquilas y contentas en la casa que alquilamos", lo cual resalta como dinamizó su red familiar para proteger y cuidar a sus hijos, quienes "están bien", dando cuenta de procesos relacionales que los involucran a todos.

\section{Proceso de subjetivación}

De poco poder personal y temor al control y al castigo del Estado a recuperación del poder para ser madre que protege y cuida, con capacidad de decidir.

Historias. La madre como cuidadora se siente culpable, con incertidumbre (348-CIN-M1) por la posible interpretación que pueda dar el sistema de control del estado a la enfermedad del hijo. Se establece una paradoja en la construcción de sentido: la madre lleva al hijo al hospital buscando su mejoría, pero la demanda constante de los servicios de salud puede ser interpretada por el ICBF como consecuencia de su falta de cuidado $(178,224-\mathrm{ECl}-$ м1). Esto genera inseguridad, temor a ser juzgada y autocuestionamiento de su rol de madre, desde la incapacidad para cuidar y proteger en la cual esta encerrada.

Memorias. Al conversar sobre esta condición y reflexionar respecto a ser madre, se narra a sí misma cómo capaz de proteger, siendo así, sus consultas repetidas al sistema de salud las asume como una acción desde su criterio para decidir y actuar cuando su hijo está en riesgo, como lo prioritario, respecto a ser una carga para los profesionales de la salud:

236-CIN-m1: Yo le digo (al esposo) prefiero ser siete hospitales y no que el niño por ahí se me empeore o se me muera acá en la casa. Sé que, si voy y lo llevo a un hospital, el hospital le pone oxígeno [...] o le hace algo y no lo veo yo morir en la casa $[. .$.

Relatos alternos. Ante la pregunta: ¿Cómo se ve frente al ICBF?, manifiesta que le ha tocado aprender de los errores, por lo tanto, ha estado dispuesta a generar cambios en sus formas de ser madre para no volver a cometerlos, teniendo en cuenta las edades y circunstancias de los niños.

Frente a: ¿Con que cuenta para el futuro? Se narra desde el "sueño de trabajar" y sentirse productiva si su hijo esta bien y no requiere ir al hospital. Se muestra como una mujer con capacidad de afrontar las dificultades, una madre que puede enfrentar el miedo y es capaz cuidar a sus hijos.

Para los profesionales, al observar su ejercicio profesional centrado en lo biológico, emerge la inquietud respecto al cambio que pueden llevar a cabo desde sí mismos: 89-CINPro-DJ: “[...] pero queda la inquietud propia del tema, es muy importante y lo pienso a manera personal, o sea ¿qué más estoy haciendo?, y con lo poco que tenemos y las limitaciones que tenemos, que son evidentes, decir “¿qué podemos desarrollar?". Surgen a partir de esta conversación nuevas formas de organización para integrar a la intervención los procesos emocionales y relacionales con los pacientes.

\section{Ciclo Adolescencia}

Paciente de 16 años remitido de un hospital de primer nivel por presentar un cuadro clínico de tres meses de evolución, con impresiones diagnósticas de: "Ictericia e Hiperbilirrubinemia de origen indirecto" con antecedentes de relaciones sexuales sin protección. Ordenan paraclínicos para explorar origen de la ictericia y los exámenes de rutina 
desde la norma técnica, solicitados a jóvenes sexualmente activos.

\section{Emergencia de bienestar en el contexto de salud en la relación paciente-familia-equipo profesional}

\section{Acoples relacionales de los miembros de la familia y del sistema de salud alrededor del cuidado}

Historias. A la indicación de exámenes que incluyen Elisa VIH, VDRL para explorar infecciones de transmisión sexual, madre e hijo asumen con temor la posibilidad de haber contraído una de estas, frente a la pregunta: ¿Cómo asumen el joven y la madre el diagnóstico: la madre responde: "Yo me asusté porque me dije: ¿y por qué le mandan esos exámenes... porque dijo que había tenido relaciones sexuales sin condón?" (140-CA-M). El joven, expectante respecto a la opinión médica, no pregunta por los riesgos, aunque manifiesta sentir temor.

La responsabilidad de cuidar, asignada por la familia y de activar la red de apoyo del sistema de salud, es de la madre. Apropiada de lo que considera su rol, (87-CA-M) ve al padre, como quien dice que hacer, pero que no cumple lo que le corresponde como miembro de la familia en esta situación: "Bueno, como él (esposo) dice que entonces hay que traerlo al médico y que según lo que le digan los médicos, pero ya... no es como, pongámosle... él debería estar con nosotros acá" (91-CA-M).

Memorias. Al dialogar surge que en la relación todos los miembros de la familia pueden ser responsables del cuidado de la salud y que los hijos pueden ejercer autonomía responsable de su propio cuidado como parte de su crecimiento.

Relatos alternos. Se replantean, los espacios asistenciales asociados al diagnóstico y manejo médico a partir de la experiencia vivida por el joven y la madre, donde es fundamental brindar el contexto para que pacientes y familia expongan sus inquietudes, temores y necesidades. El inicio de relaciones sexuales en el joven y sus implicaciones en la salud se convierte en un evento que integra la relación paternofilial respecto a los límites, autonomía y responsabilidades de los diferentes miembros de la familia. Asiímismo, demanda de los profesionales posicionamientos de corresponsabilidad respecto a la orientación en el cuidado de la salud, lo cual da cuenta de estados de bienestar construidos conjuntamente.

\section{Procesos de subjetivación}

La madre asume responsabilidad por el riesgo en la salud de su hijo, pero se reconoce la importancia del joven desarrollar la capacidad de dar cuenta de su propio cuidado con autonomía y responsabilidad.

Historias. El adolescente acata las decisiones de la madre respecto a los procesos a seguir, deriva la preocupación por su síntoma a esta, no da cuenta de su propio cuidado. La madre vive desde el sufrimiento e incertidumbre el riesgo de enfermedad crónica en su hijo.

La consulta médica alerta respecto a las situaciones que podrían limitar la capacidad del joven para desarrollar sus proyectos vitales.

Memorias. El adolescente se asume generativamente como un sujeto con capacidad de adoptar una postura crítica y reflexiva al decidir en quien confiar sus asuntos personales, actuando con sinceridad frente al médico y su madre para el manejo de su salud.

Relatos Alternos. La madre se reconfigura identitariamente para dar lugar a un hijo autónomo y responsable, con capacidad para visualizar el futuro.

Frente a la pregunta: ¿Cómo puede cuidar de si?: el joven reconoce sus recursos y fortalezas para un afrontamiento generativo frente al posible diagnóstico: "Pues aclaré que una enfermedad no le marca la vida a uno y hoy estoy seguro de [...] poder conversar con muchas personas para decidir sobre mi salud" (701-CA-D).

Emerge un joven capaz de enfrentar dificultades a partir de asumirse responsable de su cuidado y los riesgos para su salud, con las competencias 
necesarias para plantear el diálogo como elemento fundamental de la confianza en la relación médicopaciente, asociado al deber ser del acto médico ético, guiado en el derecho del paciente a ser informado acerca de lo que tiene, y a que se le resuelvan sus expectativas (277, 278-CA).

\section{Ciclo Adulto}

Hombre de 34 años, técnico de computadores, casado, con cuatro hijos en edades entre un mes de nacido y 15 años; con "diabetes mellitus" diagnosticada hace un año e "hipertensión arterial" diagnosticada hace 10 años y "obesidad". Hospitalizado 15 días antes, por descompensación de su diabetes asociada a infección de vías urinarias. Carácter hereditario de hipertensión arterial, diabetes y obesidad (madre, padre y hermanos).

\section{Emergencia de bienestar en el contexto de salud en la relación paciente-familiar-equipo profesional.}

\section{Acoples relacionales de los miembros de la familia y del sistema de salud alrededor del cuidado.}

Historias. Los diferentes diagnósticos dados por el sistema de salud y los antecedentes familiares no logran generar cambios en los hábitos que ponen en riesgo su salud dentro de las prácticas de la familia.

302-CAD-G: [...] si yo llego y me dan pan con chocolate ipues pan y chocolate! Lo que me den, pero nunca hemos estado así de "tratemos de hacer esto", o "podemos cocinar esto, o procuremos no incluir en nuestra alimentación esto, o menos gaseosas".

Las condiciones administrativas del sistema de salud que no permiten la oportuna asignación de citas médicas, son vividas por el paciente como un obstáculo para que no asuma con mayor responsabilidad su cuidado, contexto donde la atención se centra fundamentalmente en lo biológico para el restablecimiento de su salud. La asistencia a los servicios de salud hace parte de una relación obligada cuando la enfermedad evoluciona a situaciones de gravedad. En la hospitalización actual, centra el malestar en la distancia de su núcleo familiar (216, 308-CAD-G).

Las formas de relación que dan cuenta del autocuidado se organizan como imposición/indiferencia ante las condiciones del contexto: la alimentación que le sirvan, la disponibilidad de citas, el sedentarismo debido a su trabajo, la dificultad para encontrar comida saludable, entre otros.

Memorias. En el diálogo, los cambios biológicos se asocian a estilos de vida, adherencia al tratamiento y formas de relación al interior de la familia, compartiendo más tiempo con su esposa e hijos, dar afecto y proteger, con desempeño laboral que permite responder económicamente por su familia; aspectos que facilitan que la experiencia de enfermedad y su cuidado permeen generativamente la dimensión emocional.

Relatos Alternos. El paciente reconoce su responsabilidad en cuanto al autocuidado independiente de las condiciones administrativas, asumiendo que la enfermedad crónica no solo se relaciona con la salud física, sino que tiene implicaciones relacionales que impregnan la dimensión familiar en cuanto a sus formas de vida. Al respecto su hija afirma: "Lo que dice mi papá es verdad, porque sin salud no puede, él dice que quiere estar pendiente de nosotros, pero sin salud..." (258-CAD-Y).

Surge la posibilidad de que haya bienestar para todos los miembros de la familia, liderado este proceso por el padre, quien es responsable de su propio cuidado y del cuidado de los otros que dependen de él.

\section{Procesos de subjetivación}

Construcción de identidad desde la imposibilidad de autocuidado. El padre en un ejercicio de libertad responsable asume a su familia alrededor de la prevención y el cuidado.

Historias. La dimensión biológica no ha permeado el sí mismo para la responsabilidad por el cuidado propio y de su familia. 
Memorias. Generativamente, el sujeto relacionalemocional se articula en el ser un padre que extraña a su familia durante la hospitalización, que cuida de su vida y que tiene la responsabilidad de proveer afectiva y económicamente, como también enseñar a sus hijos la importancia del autocuidado.

306-CAD-G: "uno no es consciente de que estas enfermedades son de alto riesgo y de pronto uno no mira el problema a fondo y no mide las consecuencias que puede tener, todo sigue normal y de pronto uno no se interesa a buscar ayuda..."

(235-CAD-G): [...] de pronto los hábitos que llevaba, un mal ejemplo para ellos con comidas que no deben ser, como gaseosas o los tamales ¿si me entiende? (100-CAD-G)

La experiencia de salud-enfermedad asociada al diagnóstico y el cuidado mutuo con un sentido generativo, posibilita que surjan reorganizaciones para sí mismo y para la familia, el conversar en pareja y llegar a acuerdos en estilos de vida saludable, posibilita ser padres con responsabilidad compartida (298, 299, 300, 302-CAD).

Relatos Alternos. Emerge un sujeto con opción de decidir y con alternativas de solución con los recursos que posee $(235,238$-CAD-G), asume su responsabilidad en el cuidado y en la evolución de la enfermedad:

De pronto uno mismo poner de su parte ¿no?, porque si hago lo que hice al principio que vine una o dos veces y no volví, también es un error que yo estoy cometiendo..., entonces primero que todo yo concientizarme que uno realmente necesita la ayuda y necesita cuidarse (245-CAD-G).

Siete meses después se hizo un seguimiento al paciente, quien frente a: "los logros alcanzados" manifestó:

“El dialogo me sirvió 'para reflexionar' y poder asumir mi propio cuidado de mis hábitos, pues ya sé que soy yo el que tengo que cuidarme". Desde ese momento no ha vuelto a tener ninguna agudización y dice: "mi familia está mucho más tranquila".

\section{Ciclo Adulto Mayor}

Mujer de 85 años, quien ha tenido varias y largas hospitalizaciones y ha requerido cuidados domiciliarios, con diagnósticos de "enfermedad pulmonar obstructiva crónica e insuficiencia renal crónica", en estado terminal.

\section{Emergencia de bienestar en el contexto de salud en la relación paciente-familia-equipo profesional.}

\section{Acoples relacionales de los miembros de la familia y del sistema de salud alrededor del cuidado.}

Historias. La experiencia de salud-enfermedad gira en la hija sobre la pérdida de autonomía y cuidado permanente de la madre. Su desvinculación de otras formas de relación -consigo misma y con su familia-es vivida con malestar, impuesta, exclusiva y muy exigente, situación que no es tenida en cuenta por los hermanos (43, 44, 45, 47, 185-Сам).

Las formas de organización de la relación pacientefamilia-sistema de salud, al igual que en todas las etapas de ciclo, son configuradas por el sistema de salud en forma prescriptiva.

Memorias. La médica amplía el foco de observación en la permanencia de la paciente en el hospital a pesar de haberse dado de alta (20-CAMPROF), pues según la hija hay temor de muerte en casa por alguna complicación que no pueda asumir (166-CAM).

Se replantean dificultades en el vínculo madre-hija, reafirmadas cuando el padre muere y la hija sale del hogar; generativamente se reconfigura el contexto de cuidado para en esta fase de la enfermedad (54, 55, 56-САM), en cuanto la paciente solicita el cuidado de su hija y no de otros hijos que la habían cuidado anteriormente (54-CAM).

El miedo de la cuidadora a ser juzgada por el estado y por sus hermanos es redefinido por el nuevo valor que adquiere la relación, con un acompañamiento más claro del sistema de salud. Ahora el interés gira alrededor de acciones necesarias para 
reparar y sanar heridas; formas de afrontamiento generativo que tiene la familia para construir al final de una vida.

Relatos Alternos. La forma como se acopla la relación facilita que madre-hija adquieran bienestar no solo en requerimientos físicos y biológicos, sino asociado a los vínculos desde posturas solidarias.

En conversación con un nieto durante el seguimiento refiere que la abuela murió rodeada del apoyo y afecto de toda la familia, aproximadamente un mes después de dialogar con los investigadores/ interventores. La familia en los días siguientes se reunió, la hija-cuidadora pudo expresar sus sentimientos e ideas, lo cual fue útil para comprender y re-organizar la familia en relaciones más armoniosas y con mayor bienestar para todos.

\section{Procesos de subjetivación}

Temor al castigo social y del estado por no cuidar adecuadamente. Recuperación del poder personal y reconciliación familiar en la relación madre-hija en un cuidado solidario.

Historias. El cuidado en casa es vivido con temor por la cuidadora (176-CAM); asociado a historias en las que la muerte en casa ha sido connotada como "descuido". Se define desde el temor a ser juzgada por la familia y los sistemas de control del estado (170, 174-САМ).

Memorias. El afecto recibido del padre en la infancia posibilita que la historia familiar de desvinculación afectiva madre-hija (83-92 CAD), se redefina generativamente en una hija que aprendió a cuidar y a dar amor, (92-CAD), para sus hijos es madre amorosa, quien vive sin resentimientos. (76-САM) “[...] en el caso de mi mama se refleja es lo contrario. Ella no lo tuvo de su madre, pero nos lo brinda a nosotros."

Surge una hija que se vincula a la madre, facilitando que la enfermedad crónica terminal se reconfigure como una oportunidad para el perdón madre-hija, que sana heridas al final de la vida, experiencia que permite asumir los errores y aprender de estos (119, $120,125,126,127$-САм).
Relatos Alternos. El cuidado se reconoce como una decisión de ser hija, con renovación del vínculo afectivo (82-CAD) "[...] mientras yo pueda estar al lado de mi mama pues lo voy a hacer porque me nace".

\section{Discusión}

\section{Carácter intersubjetivo de la experiencia de salud-enfermedad y bienestar}

Para la persona diagnosticada y su familia, la enfermedad crónica es una experiencia vital, dependiente de la etapa de ciclo, que involucra sus vidas de manera integral, aportando contexto al sentido y la acción; lo que habla de la interdependencia entre las dimensiones biológica y social que propone Krieger (2017).

Gergen (2013) plantea la multiplicidad de opciones del sí mismo "self as multi-being" que emergen en las relaciones, lo cual daría cuenta del carácter diverso del self que propone Munné (2000). Este marco conceptual sirve para explicar cómo los diagnósticos médicos van más allá del órgano o sistema "enfermo". En otras palabras, las formas de relación no se circunscriben al "paciente o persona diagnosticada" limitada a la enfermedad, todo lo contrario, la enfermedad crónica, y más que la enfermedad en sí misma, el diagnóstico, permea otros dominios de las personas, ya que surgen en la interacción varias formas de ser madre, hijo o hija, pareja, etc. Vivencias que incluyen el presente, el pasado y el futuro de quien cuida o quien vive la enfermedad, asociadas a las dinámicas familiares en el contexto de salud y las relaciones que allí se estructuran conforme a las políticas del sistema. Interacciones que constituyen en el diálogo intersubjetividades.

Así, los procesos de subjetivación se plantean asociados a las relaciones paciente-cuidador-familiacontexto de salud, donde el adolescente y el adulto emergen como seres autónomos, con grados variables de dependencia respecto a los cuidadores, no así el niño por su indefensión y la persona mayor por el grado de incapacidad que representa la enfermedad crónica en este momento de la vida. 
Esta subjetividad permite asumir el propio cuidado desde una imagen de sí mismo de autonomía y de responsabilidad personal y relacional (MCNamee et al., 1999), más clara en el adulto que en el joven y menos evidente aun en el niño; sin mínimo asomo en el adulto mayor en estado terminal.

Procesos de subjetivación asociados al sentido de la vida misma de las personas en situación de enfermedad crónica y de los que cuidan, con mayor o menor autonomía y responsabilidad en cuanto a la salud y la enfermedad a partir del sentido de humanidad, donde el hombre elige su posibilidad (de cuidarse o no cuidarse) y esa elección se da en el mismo acto de crearla, siendo artífice de su devenir, lo cual está de acuerdo a los planteamientos de Heidegger (1997) y Buber (1967).

Al respecto es importante establecer lazos de conexión en cuanto al sí mismo que emerge cuando se está enfermo, experiencia que mantiene coherencia con la forma en que se viven los diversos acontecimientos en el transcurrir de la vida, y que se convierte en posibilidad de surgir desde los recursos y potencialidades que se ponen en juego en la crisis asociada al diagnóstico de la enfermedad.

\section{El tiempo en la experiencia de salud- enfermedad y su relación con la emergencia de bienestar}

El concepto de tiempo emerge como transversal a la comprensión y promoción de bienestar. La centralidad del tiempo viene dada, por una parte, en la particularidad que le confiere el ciclo vital, en relación con el sentido y significado dado a la experiencia de salud-enfermedad. Es importante destacar cómo en el momento vital, cada miembro de la familia, así como los contextos con los que se relacionan, confieren un sentido a esa experiencia, enmarcado en un tiempo experiencial singular y un tiempo institucional. Así el concepto de multitemporalidad (Prigonine, 1994) emerge como fundamental para la comprensión y promoción de bienestar, asumido este como una experiencia subjetiva, que se mueve en la confluencia de las diversas dimensiones del tiempo con sus fluctuaciones biológicas, psíquicas, sociales, culturales y familiares, en interrelación con el tiempo de las instituciones.

La etapa de ciclo vital no está ceñida exclusivamente al tiempo cronológico de la edad o al tiempo físico o determinado en razón a las enfermedades prevalentes en una etapa de la vida, se asocia además con las experiencias y en cómo se relacionan las personas: la necesidad de ser cuidado y de cuidar, o la incapacidad de autocuidado para sobrevivir y vivir con el amor de las personas significativas.

El tiempo de los acontecimientos y la experiencia da cuenta de la historia vivida que cuando se narra y se conversa sobre ella, se actualiza y adquiere un nuevo sentido, al responder a cuestionamientos como: ¿cómo es la experiencia de enfermedad crónica en el momento en que se vive? ¿cómo se cuida a quien se cuida? ¿Qué implica cuidar o no cuidar?

Así pues, el tiempo hace también referencia a un tiempo asociado al momento vital de las personas, a sus necesidades y a las circunstancias del contexto, por lo que invita a estimar los recursos o dificultades de orden económico, social o jurídico de los sistemas que exigen una serie de responsabilidades y cuidados. En ese sentido, algunos investigadores han dado cuenta de la polarización espacial étnica y económica como elementos importantes en el riesgo de presentar enfermedades como por ejemplo la hipertensión arterial (Feldman, Waterman, Coull y Krieger, 2015); lo que revela la importancia de una comprensión siempre singular y contextual de los fenómenos humanos relacionados con los conceptos de salud y enfermedad.

Así en la infancia, el hijo pequeño vive su día a día bajo el cuidado de su madre, en tanto, es el tiempo de ser madre de su hijo enfermo de forma permanente y exclusiva. Situación que puede ser vivida con temor al sentirse inadecuada en su rol (híper-vigilante a los posibles signos de la enfermedad de su hijo), observada y juzgada por los contextos social y de control del estado, mientras que en la institución de salud puede ser reconocida como una madre responsable y cuidadosa. El tiempo de la experiencia de salud-enfermedad como madre, permite un aprendizaje singular, enmarcado en un contexto cultural y social donde emerge la 
dinamización de las redes familiares como el principal recurso para el bienestar.

En la adolescencia, el joven y su madre viven un momento crítico cuando se hace el estudio del cuadro clínico, resultando borroso el límite entre salud y enfermedad, entendido borroso desde la perspectiva de Kosko (1995). Esta perspectiva permite comprender en el caso anotado, que no se puede asumir la enfermedad como un estado absoluto, aislado y excluyente, sino en relación con el estado de salud, estados uno y otro que conllevan múltiples matices. En ese sentido, en esta etapa se da paso a un acompañamiento a la madre y al hijo, donde asumen la experiencia como una alerta para la toma de responsabilidad en el inicio de la sexualidad, siendo vivido por el joven como el tiempo de la oportunidad, en tanto el cuidado de la salud posibilita aprendizajes en sus relaciones con la familia, con los pares, con los profesionales del sistema de salud y consigo mismo.

En cuanto al adulto, es el tiempo del presente donde confluyen el pasado de la inconsciencia y el descuido, y el futuro de la incertidumbre, confluencia que no da espera para iniciar su autocuidado que hoy puede convertirse en el organizador de la experiencia, así surge el hombre y el padre que responde por sí mismo y por los que dependen de él.

Para la familia de la adulta mayor es tiempo de la reconciliación, del encuentro con su hija cuidadora; tiempo del perdón y la despedida que se convierte en una ritualización del vínculo que permite el paso hacia la muerte; perdón, que como lo anotan Murillo y Salazar (2019), facilitaría la sensación de bienestar.

A su vez está el tiempo demarcado por las instituciones, un tiempo con límites y aperturas a partir de protocolos y lineamientos que definen la atención y el cuidado dentro de un sistema que institucionaliza normativamente el acceso y salida de las personas del sistema. Momentos de atención en los que se comprendió en este estudio, es esencial la apertura y la flexibilización de los profesionales para el establecimiento de relaciones humanas sensibles a las necesidades de las personas. Profesionales que, a su vez, requieren ser reconocidos en los tiempos humanos de agotamiento e impotencia por parte de las instituciones y el sistema de salud, y no exclusivamente por el tiempo del reloj que estos imponen para atender las necesidades y urgencias de los pacientes y sus familias.

Por último, el concepto de tiempo permite también entender el movimiento, el carácter dinámico de la experiencia, donde el acontecer de la vida, reta a los sistemas en su tarea evolutiva a adaptarse a cambios como son el enfermar y envejecer a partir de un momento de la vida en que se está sano o con vitalidad; se promueven entonces, en un mismo tiempo, cambios en los contextos de relación para poder co-evolucionar, en cuanto el acontecer de la vida misma lo demanda.

\section{Narrativa y diálogo generativo para la emergencia de bienestar}

En la investigación/intervención se configuran historias en relación al malestar en la experiencia de enfermedad crónica, como momentos de caos y crisis que requieren nuevas formas de organización de la relación para la adaptación y supervivencia de todos los participantes: paciente, familia y profesionales del contexto de salud; proceso que requiere conversaciones orientadas a comprender como se configura esa experiencia de malestar, temor, confusión o indiferencia ante el diagnóstico, en las cuales se favorece la emergencia de nuevas formas de sentido y acción para la evolución conjunta o co-evolución (Kauffman, 2003); enmarcado este proceso en diálogos generativos (Fried, 2015), con la intencionalidad de promover bienestar, donde el aprendizaje conlleva colaboración y apertura a nuevas versiones de la realización personal, profesional y social.

En este orden, se asume el diálogo como la posibilidad de encuentro entre los seres humanos que permite la articulación de relatos, emociones y sentires donde surgen el sí mismo y el otro, de acuerdo con lo que Echeverría (2003) define como construcción de realidades, en las que cada interlocutor es partícipe activo del mundo que vive en marcos de inteligibilidad y sentido compartidos dentro de un contexto. 
Contexto que da cuenta del bienestar humano desde una perspectiva generativa, construido conjuntamente en relación con los procesos de coevolución y desarrollo en términos de la capacidad de las personas para alcanzar formas de vida, que de acuerdo a Sen (1996), implican "combinaciones alternativas que una persona puede hacer o ser: los distintos funcionamientos que puede lograr" (p. 51), como tener alimentación y abrigo, pero ejerciendo la libertad y la dignidad en cuanto pueden elegir por sí mismas entre diversas opciones con los recursos disponibles para optar. Así, en el caso de la persona adulta mayor, vive la experiencia de la etapa final de la vida y la preparación para su muerte, donde su bienestar se relaciona con su capacidad de optar por la reconciliación con su hija, quien comprometida con su cuidado brinda un contexto de dignidad y respeto.

Se relaciona entonces el bienestar con la capacidad de actuar para lograr metas personales en el contexto de las relaciones significativas, como cuando los pacientes y cuidadores logran mayores niveles de autonomía y obran más por decisión propia que por miedo o adopción de una posición sumisa con los contextos de salud o de control del estado, todo esto en un marco de responsabilidad relacional donde se trasciende a la solidaridad y al valor del otro en su humanidad.

Ahora bien, en el caso específico, el bienestar es parte de los procesos de subjetivación, en tanto la persona se asume con la capacidad para cuidarse y cuidar, para responder por sus actos, para perdonar y reconciliarse con sus familiares, proceso que da cuenta de autonomía al alcanzar bienestar desde las diversas dimensiones del sí mismo que emerge en el acto de narrar y ser narrado: cuando se pide ayuda, cuando se brinda protección y cuidado, cuando se acepta la decisión de la persona enferma de seguir o no seguir una prescripción médica, cuando se da orientación y cuando se reconoce la capacidad humana de afrontar los momentos críticos que implican aceptación del diagnóstico de la enfermedad.

Es importante en este punto, resignificar el concepto de autonomía. Se acepta la importancia de fomentar la capacidad de gestión de las personas en los procesos de salud-enfermedad, esta idea requiere algunas precisiones. En los extremos de la vida, infancia y adulto mayor en estado terminal, se evidencia que la autonomía es potestad de los cuidadores para la emergencia del bienestar, pero en la experiencia misma del paciente, el asumirse como plantea Pelluchon, (2015) en su propuesta de una Ética de la Vulnerabilidad; como frágil, necesitado de ser acompañado atendido, arropado y sostenido por los cuidadores, familiares o profesionales, trasciende para el bienestar la idea de autonomía. El bienestar del paciente en estos extremos se traduce en una relación amorosa de aceptación y cuidado.

\section{Conclusiones}

En el diálogo generativo y la reflexión que se promueve en los encuentros con pacientes, familias, profesionales e investigadores/interventores, con la intencionalidad de promover bienestar en experiencias de salud-enfermedad crónica, se construyen formas particulares de relación que permiten a las personas reconfigurar su experiencia, ampliar el panorama de posibilidades y negociar significados, entre otros. Esto implica desbordar las predicciones y los determinismos que han definido la enfermedad crónica y su abordaje desde el modelo biomédico para adentrarnos en las posibilidades de las lecturas que convocan diversos campos disciplinares en interlocución con quienes viven la enfermedad, conjuntamente con sus familias, para construir nuevas versiones de la salud y la enfermedad, donde el bienestar sea una emergencia que construyen conjuntamente y que les beneficia en un ejercicio colaborativo y de corresponsabilidad.

\section{Referencias}

Anderson, H. (1997). Conversación, Lenguaje y Posibilidades. Un enfoque Posmoderno de la Terapia. Buenos Aires, Colombia: Amorrortu Editores.

Buber, M. (1967). ¿Qué es el hombre?. Ciudad de México: Fondo de Cultura Económica.

Corbin, J. y Strauss, A. (2010). Bases de la investigación cualitativa. Técnicas y procedimientos para la teoría fundamentada (Primera edición en español). Medellín: Universidad de Antioquia. 
Echeverría, R. (2003). Ontología del Lenguaje (Sexta). Santiago de Chile: Comunicaciones Noreste.

Estupinán, J. y González, O. (2012). Narrativa conversacional, relatos de vida y tramas humanos. Bogotá: Edicioes USTA.

Feldman, J., Waterman, P., Coull, B. y Krieger, N. (2015). Spatial social polarisation: using the Index of Concentration at the Extremes jointly for income and race/ethnicity to analyse risk of hypertension. Journal of Epidemiology and Community Health, 69, 1199-1207. https://doi. org/10.1136/jech-2015-205728 1199

Fried, D. (2010a). Pespectiva generativa en la gestión de conflictos sociales. Revista de Estudios Sociales, 36, 51-63.

Fried, D. (2010b). Procesos generativos en el diálogo: complejidad, emergencia y auto-organización. Plumilla Educativa, 7(1), 61-73. https:// doi.org/10.30554/plumillaedu.7.533.2010

Fried, D. (2013). Prácticas dialógicas generativas en el trabajo con familias. Revista Latinoamericana de Estudios de Familia, 5, 127-159.

Fried, D. (2015). Diálogos para la transformación. Experiencias en terapia y otras intervenciones psicosociales en Iberoamérica. WorldShareBooks.

Garzón, D. y Rojas-Gil, M. (2016). Emergencia de bienestar en enfermedades crónicas: una aproximación conceptual. Revista de Salud Pública, 18(6), 996-1004. https://doi.org/10.15446/rsap.v18n6.55278

Gergen, K. (1996). Realidades y relaciones. Aproximaciones a la construcción social.

Gergen, K. (2013). Relating with self and others. Interaction, 5(1), 9.25.

Heidegger, M. (1997). Ser y tiempo. Santiago de Chile: Editorial Universitaria.

Higuera-Dagovett, E., Rojas-Gil, P. y Garzón, D. (2015). Experiencia de hipertensión arterial en la relación paciente, familia y contexto de ayuda. Revista de Salud Pública, 17(6), 878-855. https://doi.org/10.15446/rsap.v17n6.49228

Higuera-Dagovett, E y Garzón, D. (2019). Posturas de autocuidado, relaciones y significado de la hipertensión arterial. Revista Cubana de Medicina General Integral, 35(2), 1-3.

Kauffman, S. (2003). Investigaciones. Complejidad, autoorganización y nuevas leyes para una biología general. Barcelona: Tusquets Editores.

Kosko, B. (1995). Pensamiento borroso. Barcelona: Grijalbo-Monsasori.

Krieger, N. (2005). Embodiment: a conceptual glossary for epidemiology. Journal of Epidemiology and Community Health, 59, 530-355. https:// doi.org/10.1136/jech.2004.024562

Krieger, N. (2017). Health Equity and the Fallacy of Treating Causes of Population Health as if They Sum to $100 \%$. American Journal of Publica Health, 107(4), 541-548. https://doi. org/10.2105/ AJPH.2017.303655)

McNamee, S., Gergen, K., Anderson, H., Burkitt, I., Cooperrider, D. ... Cottor, R. (1999). Relational Responsibility. Resources for Sustainable Dialogue (K. McNamee, S., Gergen, ed.). Thousand Oaks: SAGE Publications.

Munné, F. (2000). El self paradójico: la identidad como substrato del self. In La mirada psicosociológica. Grupos, procesos, lenguajes y culturas (pp. 743-749). Madrid: Biblioteca Nueva.

Murillo, J. y Salazar, M. (2019). La autoestima, la extraversión y el resentimiento como variables predictoras del bienestar subjetivo. Universitas Psychologica, 18(1), 1-18. https:// doi.org/10.11144/Javeriana.upsy 18-1.aerv

Pakman, M. (1995). Investigación e Intervención en grupos familiares. Una perspectiva constructivista. En J. Delgado y J. Gutiérrez (Eds.), Métodos y técnicas cualitativas de investigación en ciencias sociales (pp. 359-377). Madrid: Síntesis. 
Pelluchon, C. (2015). Elementos para una ética de la vulnerabilidad. Bogotá: Editorial Pontificia Universidad Javeriana.

Prigonine, I. (1994). "De los relojes a las nubes" (S. Fried, ed.). Buenos Aires: Paidós.

Sen, A. (1996). Capacidad y Bienestar. En M. Sen, A. y Nussbaum (Ed.), La Calidad de Vida. Ciudad de México: Fondo de Cultura Económica.
Varela, F. (2002). El fenómeno de la vida (Segunda ed). Santiago de Chile: Dolmen Ediciones.

Von Foerster, H. (1996). Las semillas de la cibernética. Obras escogidas de Heinz Von Foerster. Barcelona: Gedisa.

White, M. y Epston, D. (1993). Medios narrativos para fines terapéuticos. 